RESEARCH ARTICLE

\title{
Up conversion and excited state absorption analysis in the Tm:YAG disk laser multi-pass pumped by $1 \mu \mathrm{m}$ laser
}

\author{
Enmao Song ${ }^{1,2}$, Guangzhi Zhu ${ }^{1,2}$, Hailin Wang ${ }^{1,2}$, Hantian Chen ${ }^{1,2}$, Yefeng Qian $^{1,2}$, Kozlov Aleksei $^{3}$, and \\ Xiao Zhu ${ }^{1,2}$ \\ ${ }^{1}$ School of Optical and Electronic Information, Huazhong University of Science and Technology, Wuhan 430074, China \\ ${ }^{2}$ National Engineering Research Center for Laser Processing, Wuhan 430074, China \\ ${ }^{3}$ POLYUS Research Institute of M.F. Stelmakh Joint Stock Company, Moscow 117342, Russia \\ (Received 11 September 2020; revised 7 November 2020; accepted 24 December 2020)
}

\begin{abstract}
We present a $2 \mu \mathrm{m}$ Tm:YAG disk laser multi-pass pumped by a $1 \mu \mathrm{m}$ laser. The transitions in competition of up conversion (UC) and excited state absorption (ESA) are analyzed in detail based on a numerical model that considers stimulated emission, fluorescence, non-radiative decay, UC, and ESA, showing good agreement with experiments. The proportions and fractional thermal loads of all transitions are derived quantitatively. The results show that UC and ESA are critical in Tm:YAG disk lasers, resulting in a decrease in the absorbed pump power and temperature after lasing for two different disk thicknesses. In addition, although the absorbed pump power of the $0.5 \mathrm{~mm}$ disk is lower than in the $1 \mathrm{~mm}$ case, its relatively weak UC and ESA and low total fractional thermal load can improve laser performance. A $0.5 \mathrm{~mm}$ Tm:YAG disk laser thus delivered the maximum output power of $1.05 \mathrm{~W}$ with beam quality of $M_{x}^{2}=2.02$ and $M_{y}^{2}=2.03$.
\end{abstract}

Keywords: mid-infrared laser; thermal effects; thulium

\section{Introduction}

Tm:YAG lasers operating at $2 \mu \mathrm{m}$ wavelengths have attracted a lot of attention owing to their strong absorption in water, as well as the eye-safe wavelength bands, which have been utilized extensively in soft-material processing, medical diagnostics, direct optical communications, and so on $^{[1-3]}$. One of the main challenges is related to the induced laser performance degeneration that arises from waste heat generated inside the crystal, which is detrimental to the cited applications. Increasing the heat dissipation capability and analyzing thermal problems in Tm:YAG lasers are thus critical for laser developers.

Efficient heat removal is realized by increasing the surfaceto-volume ratio of the gain medium, as in fiber, slab, and disk lasers ${ }^{[4-7]}$. Among them, disk architectures have several advantages, including efficient cooling capacity, weak nonlinear effect, and power scaling without compromising

Correspondence to: G. Zhu, School of Optical and Electronic Information, Huazhong University of Science and Technology, Wuhan 430074, China. Email: zgzlaser@hust.edu.cn beam quality ${ }^{[8]}$. The highest published output power of a Tm:YAG disk laser is $24 \mathrm{~W}$ with $M^{2}$ value of 11.7 and slope efficiency of $31 \%$ when pumped at $785 \mathrm{~nm}$, in which the presence of cross-relaxation (CR) can increase the pump quantum efficiency ${ }^{[9,10]}$. In addition, the use of a $1 \mu \mathrm{m}$ laser as the pump source has also proved successful in generating $2 \mu \mathrm{m}$ radiation ${ }^{[11]}$. This is a readily available and costeffective pump method, because the $1 \mu \mathrm{m}$ laser (e.g., lamppumped $\mathrm{Nd}$ :YAG laser, $\mathrm{Yb}^{3+}$ doped laser) is commercially mature with high power, high reliability, and mass production. Very recently, an intra-cavity pumped Tm:YAG disk laser in multi-transverse mode operation was reported with an average output power of $760 \mathrm{~mW}$ in quasi-continuous wave mode and $17 \mathrm{~mW}$ in continuous wave mode ${ }^{[12]}$. The relatively low absorption coefficient of $1 \mu \mathrm{m}$ laser for a disk-shaped gain medium is compensated by the intra-cavity pumping method ${ }^{[11]}$. Nevertheless, its poor pumping stability and strong nonlinear coupling can degrade the output laser performances significantly ${ }^{[13,14]}$. It is then difficult to study the operation characteristics of $1 \mu \mathrm{m}$ pumped Tm:YAG disk laser in detail. 
On the other hand, because a high population inversion is necessary to compensate for the limitation of the disk's thickness, the intensities of transitions in competition, up conversion (UC) and excited state absorption (ESA), cannot be ignored, as is done in rod and fiber-shaped lasers ${ }^{[4]}$. The enhancement of output power is then limited and more heat is generated in Tm:YAG disk lasers. Therefore, verifying the proportions of all transitions and evaluating their effects on operation characteristics and heat generation are extremely important for Tm:YAG disk lasers.

In the present paper, a dual parabolic mirror 20-pass pumping scheme is designed for the $1 \mu \mathrm{m}$ pumped Tm:YAG disk laser. The multi-pass pumping method ensures high pumping stability and efficient thermal management of the gain medium ${ }^{[15]}$. This scheme can also enable the measurement of the unabsorbed pump power in real time. The absorption properties of $1 \mu \mathrm{m}$ pump light are then studied efficiently. In addition, a numerical model considering stimulated emission (SE), fluorescence, non-radiative decay, $\mathrm{UC}$, and ESA is developed to evaluate the proportions and fractional thermal loads of all transitions quantitatively in view of the absorption, output, and temperature properties in this laser. Experimental and theoretical results show that the UC, ESA, and SE compete with each other to consume the $1 \mu \mathrm{m}$ pump photons, and the proportions of $\mathrm{UC}$ and ESA are more dominant than that of SE in a $1 \mu \mathrm{m}$ pumped Tm:YAG disk laser, leading the absorbed pump power to decrease abnormally after lasing. The strong UC and ESA effects are the main part of total heat generation for two thicknesses of gain medium. In addition, the small thickness of gain medium with better heat dissipation capacity has relatively weak UC and ESA and low total fractional thermal load, which can improve the output laser performances. Consequently, a $0.5 \mathrm{~mm}$ Tm:YAG disk laser delivered an average power of $1.05 \mathrm{~W}$ with slope efficiency of $13.2 \%$, and the measurement of it yielded $M_{x}^{2}=2.02$ and $M_{y}^{2}=2.03$.

\section{Theoretical analysis}

The energy diagram of a Tm:YAG crystal is shown in Figure 1, where $\lambda_{P}$ and $\lambda_{L}$ are the wavelengths of the pump and output laser, respectively.

The $\mathrm{Tm}^{3+}$ ions in the ${ }^{3} \mathrm{H}_{6}$ state can be promoted to the energy level near the ${ }^{3} \mathrm{H}_{5}$ state by stimulated absorption (SA), followed by decaying to the ${ }^{3} \mathrm{~F}_{4}$ state via non-radiative transition and CR, thereby lasing at $2 \mu \mathrm{m}$ and emitting fluorescence. Here $\lambda_{F}^{3 F 4}$ is the average fluorescence wavelength from the ${ }^{3} \mathrm{~F}_{4}$ to ${ }^{3} \mathrm{H}_{6}$ state and is given by

$$
\lambda_{F}^{3 F 4}=\frac{\int \varepsilon(\lambda) \lambda \mathrm{d} \lambda}{\int \varepsilon(\lambda) \mathrm{d} \lambda},
$$

where $\lambda$ is the wavelength and $\varepsilon(\lambda)$ is the intensity at the wavelength of $\lambda$. Figure 2 shows the measured fluorescence spectrum of Tm:YAG ${ }^{[16]}$. Based on Eq. (1), the calculated average fluorescence wavelength is $1901 \mathrm{~nm}$.

In addition to $\mathrm{SE}$, there are two more competing transitions that may also deplete the population inversion of the ${ }^{3} \mathrm{~F}_{4}$ state and increase the absorption of $1 \mu \mathrm{m}$ photons, depending on the pumping density: UC and ESA. In the former case, an excited $\mathrm{Tm}^{3+}$ ion in the ${ }^{3} \mathrm{~F}_{4}$ state is promoted to the ${ }^{3} \mathrm{H}_{5}$ or ${ }^{3} \mathrm{H}_{4}$ state via energy transfer processes. In the latter case, an excited $\mathrm{Tm}^{3+}$ ion in the ${ }^{3} \mathrm{~F}_{4}$ state is promoted to the ${ }^{1} \mathrm{G}_{4}$ state by absorbing two more $1 \mu \mathrm{m}$ photons, followed by fluorescence and non-radiative decay. The fluorescence decay from the ${ }^{1} \mathrm{G}_{4}$ state will produce a broad fluorescence emission spectrum. Considering the transition rate and lifetimes of states, the equivalent fluorescence wavelength from ${ }^{1} \mathrm{G}_{4}$ to lower states $\left({ }^{3} \mathrm{~F}_{2,3},{ }^{3} \mathrm{H}_{4},{ }^{3} \mathrm{H}_{5},{ }^{3} \mathrm{~F}_{4}\right.$, and $\left.{ }^{3} \mathrm{H}_{6}\right)$ is given by

$$
\lambda_{F}^{1 G 4}=\frac{1}{\sum_{i=1}^{5} \beta_{i}^{6}\left(E_{6}-E_{i}\right)},
$$

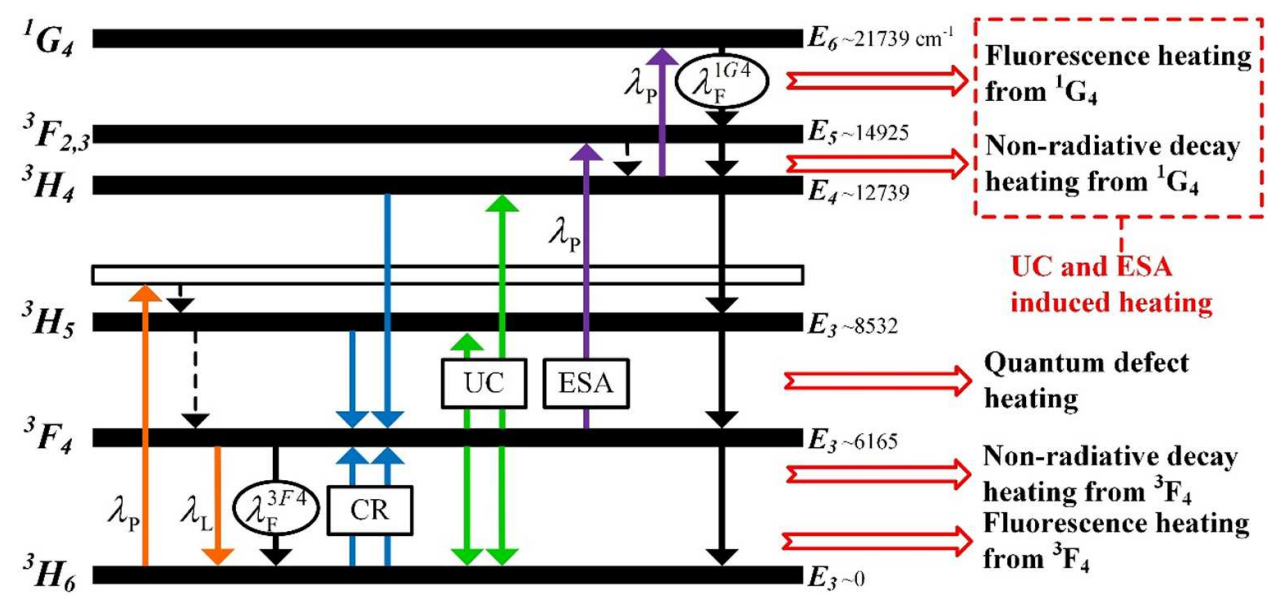

Figure 1. Energy diagram of a Tm:YAG crystal. 


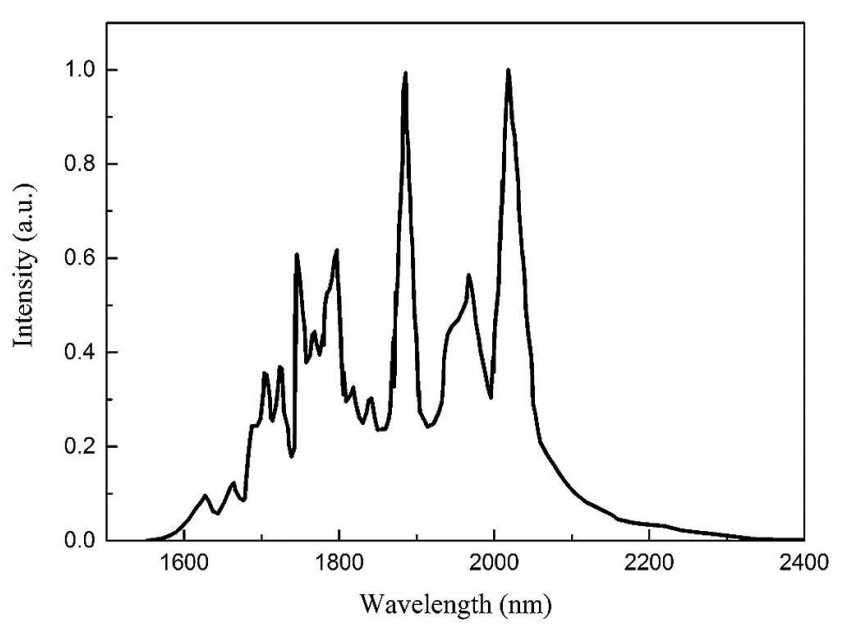

Figure 2. Fluorescence spectrum of a Tm:YAG crystal.

Table 1. The branch ratios of a Tm:YAG crystal in the ${ }^{1} \mathrm{G}_{4}$ state.

\begin{tabular}{lccc}
\hline Starting level & Ending level & Symbol & Value \\
\hline${ }^{1} \mathrm{G}_{4}$ & ${ }^{3} \mathrm{~F}_{2,3}$ & $\beta_{5}^{6}$ & 0.030 \\
${ }^{1} \mathrm{G}_{4}$ & ${ }^{3} \mathrm{H}_{4}$ & $\beta_{4}^{6}$ & 0.092 \\
${ }^{1} \mathrm{G}_{4}$ & ${ }^{3} \mathrm{H}_{5}$ & $\beta_{3}^{6}$ & 0.375 \\
${ }^{1} \mathrm{G}_{4}$ & ${ }^{3} \mathrm{~F}_{4}$ & $\beta_{2}^{6}$ & 0.131 \\
${ }^{1} \mathrm{G}_{4}$ & ${ }^{3} \mathrm{H}_{6}$ & $\beta_{1}^{6}$ & 0.373 \\
\hline
\end{tabular}

where $E_{i}(i=1,2,3,4,5)$ is the energy for each state and $\beta_{i}^{6}$ is the fluorescence branch ratio for ${ }^{1} \mathrm{G}_{4}$ state, as shown in Table $1^{[17]}$. As the fluorescence branch ratio is related to the transition rate and lifetimes of the lower states, its influences have been taken into account in the model ${ }^{[18]}$. In addition, the ${ }^{3} \mathrm{~F}_{2,3},{ }^{3} \mathrm{H}_{4}$, and ${ }^{3} \mathrm{H}_{5}$ states have very short lifetimes and low quantum efficiency, which can make the $\lambda_{F}^{1 G 4}$ blueshifted. As a result, the equivalent fluorescence wavelength is calculated as $649.7 \mathrm{~nm}$.

In Figure 1, the processes of heat generation in a Tm:YAG crystal could be divided into the following four parts: quantum defect heating, non-radiative decay heating from the ${ }^{3} \mathrm{~F}_{4}$ state, fluorescence heating from the ${ }^{3} \mathrm{~F}_{4}$ state, and UC- and ESA-induced heating. The heating energy density due to the quantum defect caused by SE can be described as

$$
Q_{L}=\Delta n \eta_{L}\left(h v_{P}-h v_{L}\right),
$$

where $h$ is the Planck constant, $v_{P}$ is the frequency of the pump photon, $v_{L}$ is the frequency of the laser photon, and $\Delta n$ is the density of pump photons in laser medium. Here $\eta_{L}$ is the laser extraction efficiency, which is defined as the fraction of absorbed pump photons consumed by SE.

The heat energy density generated by non-radiative decay in the ${ }^{3} \mathrm{~F}_{4}$ state can be described as

$$
Q_{N R}^{3 F 4}=\Delta n\left(1-\eta_{L}\right)\left(1-\eta_{R}^{3 F 4}\right)\left(1-\eta_{U C \& E S A}\right) h v_{P},
$$

where $\eta_{R}^{3 F 4}$ is the radiative quantum efficiency of the ${ }^{3} \mathrm{~F}_{4}$ state. Meanwhile, the heating energy density caused by fluorescence decay in the ${ }^{3} \mathrm{~F}_{4}$ state can be described as

$$
Q_{F}^{3 F 4}=\Delta n\left(1-\eta_{L}\right) \eta_{R}^{3 F 4}\left(1-\eta_{U C \& E S A}\right)\left(h v_{P}-h v_{F}^{3 F 4}\right),
$$

where $v_{F}^{3 F 4}$ is the frequency of average fluorescence wavelength from the ${ }^{3} \mathrm{~F}_{4}$ state to the ${ }^{3} \mathrm{H}_{6}$ state and $\eta_{U C \& E S A}$ is the quantum efficiency of competing transitions (UC and ESA), representing the percentage of upper-level laser particles for UC and ESA. The additional absorption efficiency is then denoted by $\left(1-\eta_{L}\right) \eta_{U C \& E S A}$, which represents the fraction of absorbed pump photons consumed by UC and ESA transitions. Here $\left(1-\eta_{L}\right)\left(1-\eta_{U C \& E S A}\right)\left(1-\eta_{R}^{3 F 4}\right)$ and $\left(1-\eta_{L}\right)\left(1-\eta_{U C \& E S A}\right) \eta_{R}^{3 F 4}$ represent the fraction of absorbed pump photons consumed by non-radiative decay and fluorescence decay in the ${ }^{3} \mathrm{~F}_{4}$ state, respectively.

The UC- and ESA-induced heating includes non-radiative decay heating from the ${ }^{1} \mathrm{G}_{4}$ state $Q_{N R}^{1 G 4}$ and fluorescence heating from the ${ }^{1} \mathrm{G}_{4}$ state $Q_{F}^{1 G 4}$, which can be described as

$$
\begin{aligned}
& Q_{N R}^{1 G 4}=\Delta n\left(1-\eta_{L}\right)\left(1-\eta_{R}^{1 G 4}\right) \eta_{U C \& E S A} h v_{P}, \\
& Q_{F}^{1 G 4}=\Delta n\left(1-\eta_{L}\right) \eta_{U C \& E S A} \eta_{R}^{1 G 4}\left(h v_{P}-h v_{F}^{1 G 4} / 3\right),
\end{aligned}
$$

where $\eta_{R}^{1 G 4}$ is the radiative quantum efficiency for the ${ }^{1} \mathrm{G}_{4}$ manifold and $v_{F}^{1 G 4}$ is the frequency of the equivalent fluorescence wavelength from the ${ }^{1} \mathrm{G}_{4}$ state.

The total fractional thermal load of a Tm:YAG crystal is given by

$$
\begin{aligned}
\eta_{h}= & \frac{\left(Q_{L}+Q_{N R}^{3 F 4}+Q_{F}^{3 F 4}+Q_{N R}^{1 G 4}+Q_{F}^{1 G 4}\right)}{\Delta n_{R} h v_{P}}=\underbrace{\eta_{L}\left(1-\frac{\lambda_{P}}{\lambda_{L}}\right)}_{\text {laser heating }} \\
& +\underbrace{\left(1-\eta_{L}\right)\left(1-\eta_{U C \& E S A}\right)\left(1-\eta_{R}^{3 F 4} \frac{\lambda_{P}}{\lambda_{F}^{3 F 4}}\right)}_{\begin{array}{c}
\text { fluorescence and non-radiative } \\
\text { decay heating in }{ }^{3} \mathrm{~F}_{4} \text { state }
\end{array}} \\
& +\underbrace{\left(1-\eta_{L}\right) \eta_{U C \& E S A}\left(1-\eta_{R}^{1 G 4} \frac{\lambda_{P}}{3 \lambda_{F}^{1 G 4}}\right)} .
\end{aligned}
$$$$
\text { UC- and ESA-induced heating }
$$

It is straightforward to show that the first term of Eq. (8) represents laser heating, the second term represents fluorescence heating and non-radiative decay in the ${ }^{3} \mathrm{~F}_{4}$ state, and the third term represents UC- and ESA-induced heating via fluorescence and non-radiative decay in the ${ }^{1} \mathrm{G}_{4}$ state. In addition, the laser extraction efficiency is given by

$$
\eta_{L}=\frac{\frac{I_{L}}{h v_{L}} g_{L} M_{R}}{\frac{I_{P}}{h v_{P}} \alpha_{P}},
$$


where $I_{P}$ is the average pump intensity in the crystal, $I_{L}$ is the power intensity of the circulating laser radiation inside the resonator, $M_{R}$ is the number of the laser passing through the crystal per resonator round trip, $\alpha_{P}$ is the absorption coefficient for pump radiation, and $g_{L}$ is the gain coefficient for laser. When the pump intensity is higher than laser threshold, the laser starts lasing and gives $g_{L} M_{R}=\delta / d$, where $d$ is the thickness of the gain medium. Here $\delta$ is the round trip loss and can be described as

$$
\delta=-\ln \left(1-T_{o c}\right)-\ln \left(1-L_{l o s s}\right)
$$

where $T_{o c}$ is the output coupler transmission and $L_{l o s s}$ accounts for residual losses, owing to the scattering during one round trip in the resonator. Equation (9) is simplified as

$$
\eta_{L}=\frac{\frac{I_{L}}{h v_{L}} \frac{\delta}{d}}{\frac{I_{P}}{h v_{P}} \alpha_{P}}=\frac{\lambda_{L}}{\lambda_{P}} \frac{I_{L} \delta}{I_{a b s}}=\frac{\lambda_{L}}{\lambda_{P}} \frac{I_{o u t} \delta}{I_{a b s} T_{o c}}
$$

where $I_{\text {out }}$ is the output power intensity and $I_{a b s}$ is the absorbed pump power intensity. When the pump intensity is lower than laser threshold, the laser extraction efficiency is zero. The total fractional thermal load is simplified as

$$
\begin{aligned}
\eta_{h}= & \left(1-\eta_{U C \& E S A}\right)\left(1-\eta_{R}^{3 F 4} \frac{\lambda_{P}}{\lambda_{F}^{3 F 4}}\right) \\
& +\eta_{U C \& E S A}\left(1-\eta_{R}^{1 G 4} \frac{\lambda_{P}}{3 \lambda_{F}^{1 G 4}}\right) .
\end{aligned}
$$

Based on the absorbed pump power $P_{a b s}$ and the total fractional thermal load $\eta_{h}$, the central temperature of Tm:YAG disk is calculated by using the finite element method. The measured temperature data are then fitted with the calculated data by adjusting $\eta_{U C \& E S A}$ and $\eta_{R}^{1 G 4}$. Consequently, the proportions and fractional thermal load of all transitions are derived. Parameters used in the simulation are listed in Table 2 .
Table 2. Basic parameters used for the model.

\begin{tabular}{lc}
\hline Parameters & Value \\
\hline Loss factor for laser in resonator $\left(L_{\text {loss }}\right)$ & $2 \%$ \\
$\mathrm{Cu}-\mathrm{W}$ plate thickness $\left(d_{C u W}\right)$ & $2 \mathrm{~mm}$ \\
Thermal conductivity of $\mathrm{Cu}-\mathrm{W}$ plate $\left(k_{C u W}\right)$ & $385 \mathrm{~W} /(\mathrm{m} \cdot \mathrm{K})$ \\
Thermal conductivity of Tm:YAG crystal $\left(k_{T m: Y A G}\right)$ & $7 \mathrm{~W} /(\mathrm{m} \cdot \mathrm{K})^{[19]}$ \\
Convective heat transfer coefficient $\left(K_{\text {heat }}\right)$ & $10 \mathrm{~W} /\left(\mathrm{m}^{2} \cdot \mathrm{K}\right)^{[20]}$ \\
Radiative quantum efficiency of ${ }^{3} \mathrm{~F}_{4}$ state $\left(\eta_{R}^{3 F 4}\right)$ & $0.98^{[21]}$ \\
Temperature of coolant fluid $\left(T_{\text {cool }}\right)$ & $292 \mathrm{~K}$ \\
\hline
\end{tabular}

\section{Experimental setup}

The experimental setup is shown in Figure 3. A multipass pumping scheme consisting of dual parabolic mirrors with a conjugated relationship is designed to send the pump beam through the disk 20 times, and the position of each pump spot on two parabolic mirrors is shown in Figure 3(a). In particular, this scheme enables the unabsorbed pump light to be emitted from the multi-pass pumping head, and it is thus beneficial to evaluate the absorption properties of $1 \mu \mathrm{m}$ pump light in this laser. In addition, the temperature of Tm:YAG disk was measured by an infrared thermal imager (Testo 890-2) with a resolution of $0.1^{\circ} \mathrm{C}$.

The Tm:YAG disk crystal was mounted on a water-cooled copper heatsink at $18^{\circ} \mathrm{C}$ with a doping concentration of $3.5 \%$, and its thickness and pump spot size were 0.5 and $1.7 \mathrm{~mm}$, respectively. In addition, the front surface of the Tm:YAG disk had an antireflection coating at both 1 and $2 \mu \mathrm{m}$ wavelengths, and the back surface had a highly reflective coating in the same wavelength band. The absorption coefficient of Tm:YAG crystal at $1 \mu \mathrm{m}$ was around $1.37 \times 10^{-3} \mathrm{~mm}^{-1}$. Moreover, the range of dioptric power of the $0.5 \mathrm{~mm}$ disk was measured as $0.1-0.93 \mathrm{~m}^{-1[22]}$. A $\mathrm{V}$-shaped cavity with large stable area was designed to match the dioptric power. M1 was a concave mirror with curvature radius of $1 \mathrm{~m}$, and it had a high reflectivity at $2 \mu \mathrm{m}$. OC1 was a plane output coupler at $2 \mu \mathrm{m}$ with a transmittance of $1 \%$, which was the optimum transmissivity by measuring the highest output power. The distances between components in
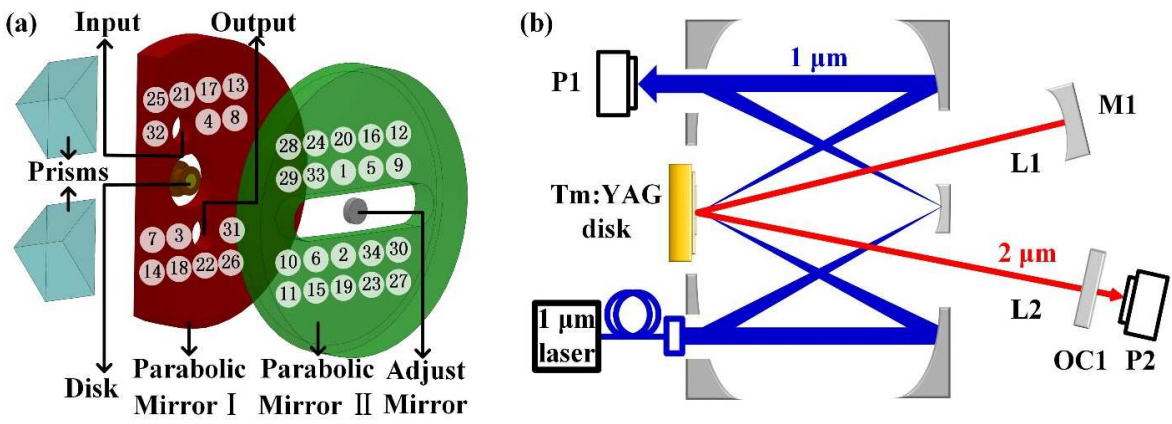

Figure 3. Layout of the (a) 20-pass pumping head and (b) $2 \mu \mathrm{m}$ Tm:YAG disk laser multi-pass pumped by a $1 \mu \mathrm{m}$ laser. 


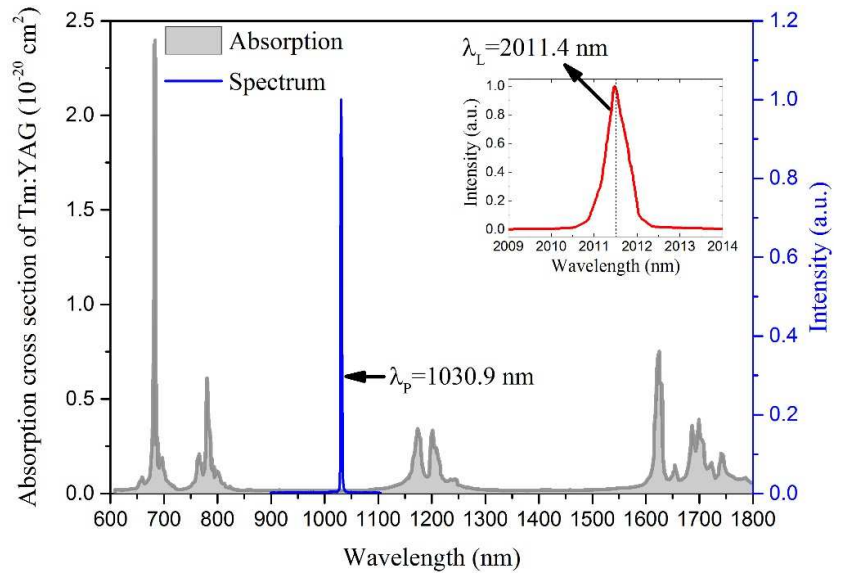

Figure 4. Absorption cross section of Tm:YAG and spectrum of pump light. Inset is the spectrum of output laser.

the cavity were $L_{1}=0.455 \mathrm{~m}$ and $L_{2}=0.25 \mathrm{~m}$. Meanwhile, a $1 \mathrm{~mm}$ thick Tm:YAG disk laser was also analyzed as a comparison, which shared the same coating parameters and doping concentration as the $0.5 \mathrm{~mm}$ disk. To realize mode matching in terms of dioptric power of the thick disk, the cavity lengths of two arms were adjusted to $L_{1}=$ $0.55 \mathrm{~m}$ and $L_{2}=0.25 \mathrm{~m}$, respectively. The number of pumping passes and pumped spot size were the same as in the $0.5 \mathrm{~mm}$ thickness case. In addition, to study the properties of absorbed pump power and output power, P1 and $\mathrm{P} 2$ were used to measure the unabsorbed pump power $P_{\text {unabs }}$ and output laser power $P_{\text {out }}$, respectively.

Actually, the pump source can be replaced by any $\mathrm{Nd}^{3+}$ or $\mathrm{Yb}^{3+}$-based laser at $\sim 1 \mu \mathrm{m}$ wavelength, as the absorption of Tm:YAG in the $1 \mu \mathrm{m}$ spectral region is almost the same (see Figure 4). The spectra of the pump light and output laser were both measured by a spectrometer (waveScan of APE), and the central wavelengths of the pump light $\lambda_{P}$ and laser radiation $\lambda_{L}$ were 1030.9 and $2011.4 \mathrm{~nm}$, respectively.

\section{Results and discussion}

During the experiments, a Yb:YAG thin disk laser was used as the pump source, and the maximum input pump power of the Tm:YAG disk laser was $P_{\text {in }}=65.8 \mathrm{~W}$. To prevent disk damage, the pump power was limited such that the disk temperature never exceeded $125^{\circ} \mathrm{C}$.

\subsection{Operation characteristics of a $1 \mu \mathrm{m}$ pumped Tm:YAG disk laser}

In practice, the reflection of $1 \mu \mathrm{m}$ laser in the multi-pass pumping scheme is incomplete ${ }^{[15]}$. Considering the loss of the multi-pass pumping scheme, the absorbed pump power

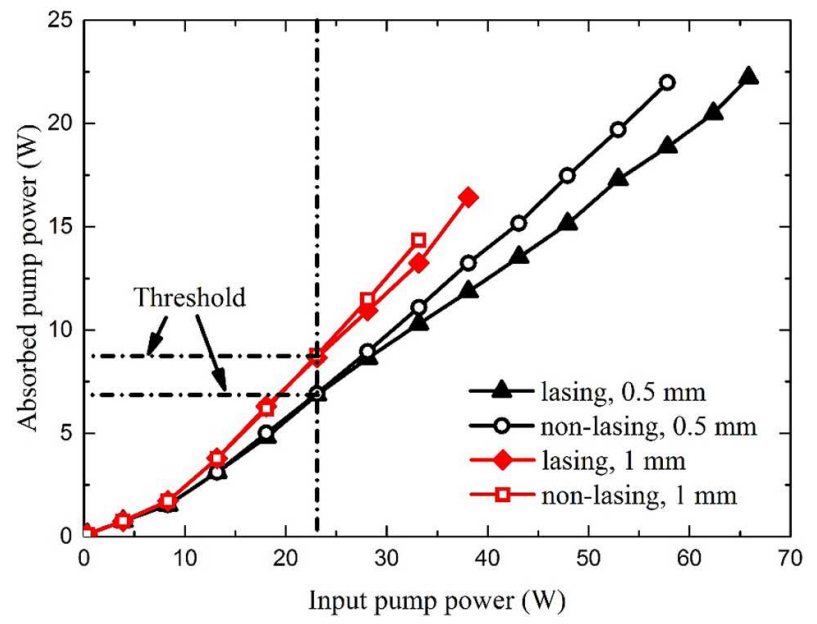

Figure 5. Absorbed pump power of a Tm:YAG crystal versus input pump power under non-lasing and lasing conditions for 0.5 and $1 \mathrm{~mm}$ thickness of gain medium.

$P_{a b s}$ is given by

$$
P_{a b s}=P_{\text {in }}-P_{\text {unabs }}-P_{\text {in }}\left(1-R_{p}\right),
$$

where $R_{p}$ is the total reflectivity of the Tm:YAG multi-pass pumping scheme with the measured value of $77 \%$.

Figure 5 shows the dependences of absorbed pump power for 0.5 and $1 \mathrm{~mm}$ Tm:YAG disk lasers on input pump power under non-lasing and lasing conditions. It can be seen that the absorbed pump power threshold of the $0.5 \mathrm{~mm}$ Tm:YAG disk laser is $6.9 \mathrm{~W}$. Above the threshold, the laser starts oscillating, and the output power increases linearly with the absorbed pump power, as shown in Figure 6. The maximum output power was $1.05 \mathrm{~W}$ with the beam quality of $M_{x}^{2}=2.02, M_{y}^{2}=2.03$, and the maximum optical-tooptical efficiency and slope efficiency were $\eta_{\text {oto }}=4.8 \%$ and $\eta_{\text {slope }}=13.2 \%$, respectively. The corresponding pump quantum efficiency was $25.8 \%$, indicating that only a small portion of the absorbed $1 \mu \mathrm{m}$ photons were used for emitting $2 \mu \mathrm{m}$ lasers. At the same time, serious red and blue light was observed in experiments, indicating that the UC and ESA were more dominant than the SE in a Tm:YAG disk laser when pumped by a $1 \mu \mathrm{m}$ laser. Compared with $0.5 \mathrm{~mm}$ disk, the $1 \mathrm{~mm}$ thick disk can absorb more pump power. At the input pump power of $38.1 \mathrm{~W}$, the absorbed pump power increases from 11.9 to $16.4 \mathrm{~W}$. However, because the performance of one-dimensional heat flow is poor for the thick-disk case, the $1 \mathrm{~mm}$ thick disk has a higher temperature than the $0.5 \mathrm{~mm}$ disk at the same absorbed pump power, and thereby a higher threshold of $8.5 \mathrm{~W}$. The output laser performances are also degraded, as presented in Figure 6.

On the other hand, the absorbed pump power of the Tm:YAG disk is significantly different under lasing and non-lasing conditions, as shown in Figure 5. Under lasing condition, the absorbed pump power of Tm:YAG decreases 
obviously for both 0.5 and $1 \mathrm{~mm}$ thicknesses of gain medium. This abnormal phenomenon is very interesting, and shows us the dynamic absorption properties of $1 \mu \mathrm{m}$ pump light for a Tm:YAG disk before and after lasing, which is equivalent to the consumption of population inversion on the upper laser level. In general, the depletion of upper laser level induces an increased absorption of pump light for a laser medium with simple energy level, e.g., Yb:YAG or Nd:YAG ${ }^{[23]}$. However, the Tm:YAG crystal shows the abnormal changes of absorbed pump power when pumped by the $1 \mu \mathrm{m}$ light. This is attributed to the significant $\mathrm{UC}$ and ESA effects. To show the operation characteristics of $1 \mu \mathrm{m}$ pumped Tm:YAG disk laser clearly, an operating principle diagram under non-lasing and lasing conditions is presented in Figure 7. The absorbed pump power of Tm:YAG mainly consists of two parts: direct absorption via SA and CR, and additional absorption via UC and ESA. Above the laser threshold, the SE, UC, and ESA compete with each other to consume the population inversion on the ${ }^{3} \mathrm{~F}_{4}$ state, and this shows the dynamic absorption properties of the pump

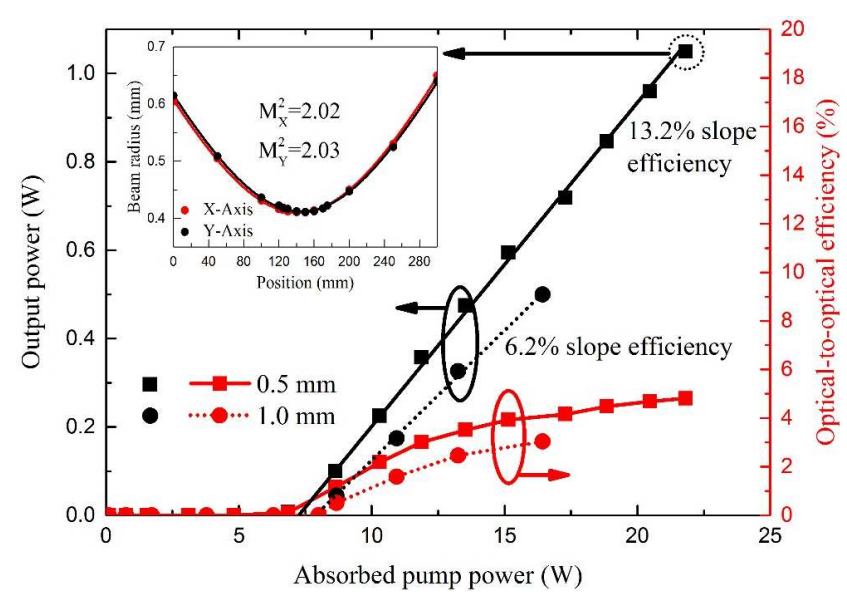

Figure 6. Output power of a Tm:YAG disk laser versus absorbed pump power for 0.5 and $1 \mathrm{~mm}$ thickness of gain medium. The inset shows the measured beam caustic and calculated beam quality at the output power of $1.05 \mathrm{~W}$. light. Under the non-lasing condition, no SE occurs, and the significant UC and ESA effects allow high absorption of the $1 \mu \mathrm{m}$ laser. At the input pump power of $57.8 \mathrm{~W}$, the absorbed pump power of the $0.5 \mathrm{~mm}$ disk was $22.5 \mathrm{~W}$. Under the lasing condition, the generation of SE makes the direct absorbed pump power increase due to the depletion of the ${ }^{3} \mathrm{~F}_{4}$ state. However, the consumption of population inversion on the ${ }^{3} \mathrm{~F}_{4}$ state can suppress the intensities of UC and ESA. The additional absorbed pump power, which is used for UC and ESA, decreases accordingly. As the intensities of UC and ESA were more dominant than that of SE, the absorbed pump power of the gain medium was decreased to $18.7 \mathrm{~W}$. On the other hand, the increased temperature of Tm:YAG is mainly caused by the UC and ESA when pumped by $1 \mu \mathrm{m}$ light, and the temperature of Tm:YAG thus drops from 121.1 to $91.1^{\circ} \mathrm{C}$ after lasing at $2 \mu \mathrm{m}$, as shown in Figure 7 .

\subsection{Evaluation intensities of UC and ESA by calculating $\eta_{U C \& E S A}$ and $\eta_{R}^{1 G 4}$}

As mentioned previously, the Tm:YAG disk laser has significant UC and ESA, which has been described qualitatively. In this section, the intensities of UC and ESA are evaluated quantitatively by calculating $\eta_{U C \& E S A}$ and $\eta_{R}^{1 G 4}$. The finite element method is used to calculate the central temperature of the gain medium on account of $P_{a b s}$ and $\eta_{h}$. The additional absorption efficiency $\eta_{U C \& E S A}$ and radiative quantum efficiency for the ${ }^{1} \mathrm{G}_{4}$ manifold $\eta_{R}^{1 G 4}$ are obtained from a leastsquares fit to the measured temperature data.

The dependences of central temperature for a $0.5 \mathrm{~mm}$ Tm:YAG disk laser on absorbed pump power are shown in Figure 8(a). When the absorbed pump power is below 0.1 $\mathrm{W}$, the power density is $<240 \mathrm{~W} / \mathrm{cm}^{3}$, which is less than the ESA threshold ${ }^{[11,12]}$. The ESA effect can then be neglected, i.e., $\eta_{U C \& E S A}=0$. When the absorbed pump power is above the ESA threshold, the best fit to data gives $\eta_{\text {UC\&ESA }}=99.6 \%$ and $\eta_{R}^{1 G 4}=0.3 \%$ for the crystal by using the finite element

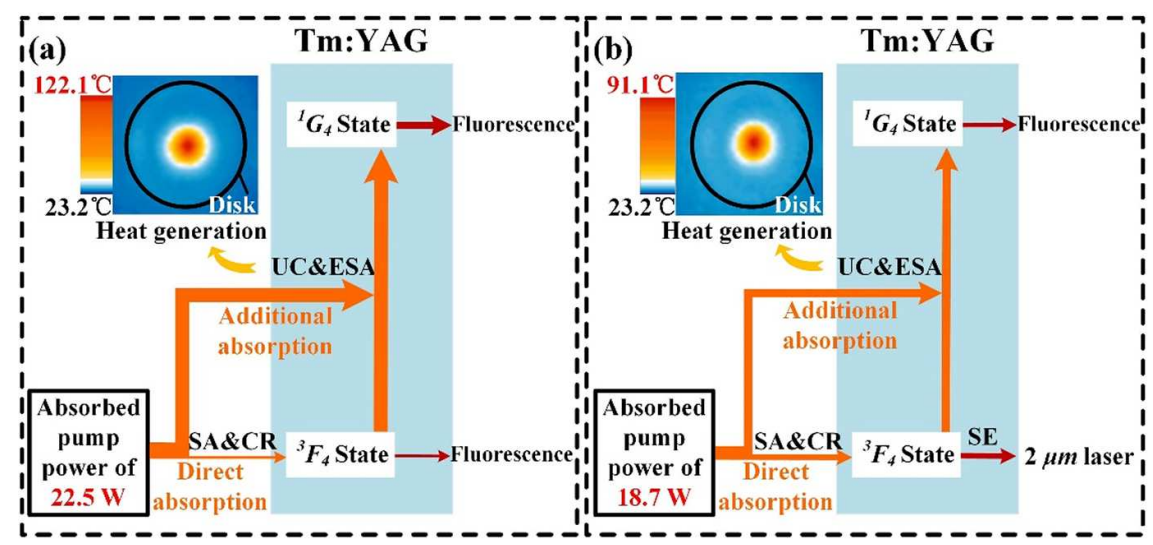

Figure 7. Operating principle diagram of the $0.5 \mathrm{~mm}$ Tm:YAG disk laser under (a) non-lasing and (b) lasing conditions. The thicknesses of the arrows are proportional to the intensities of the transitions. 
(a)

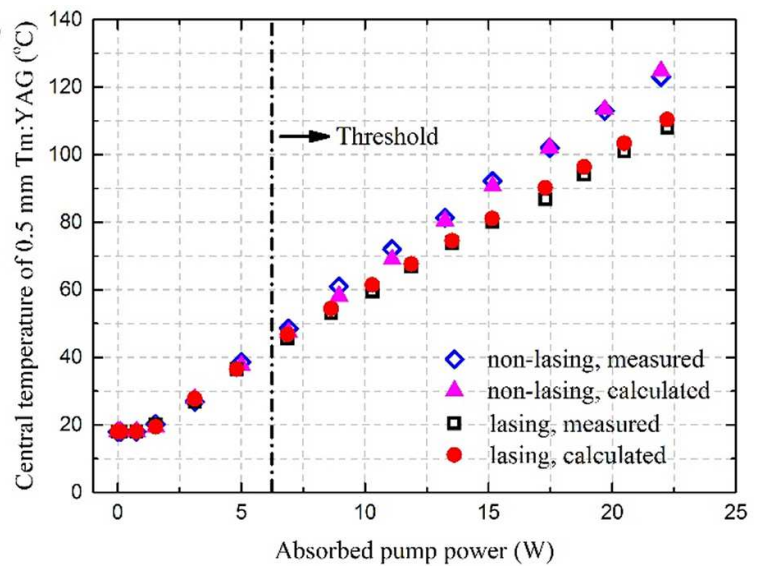

(b)

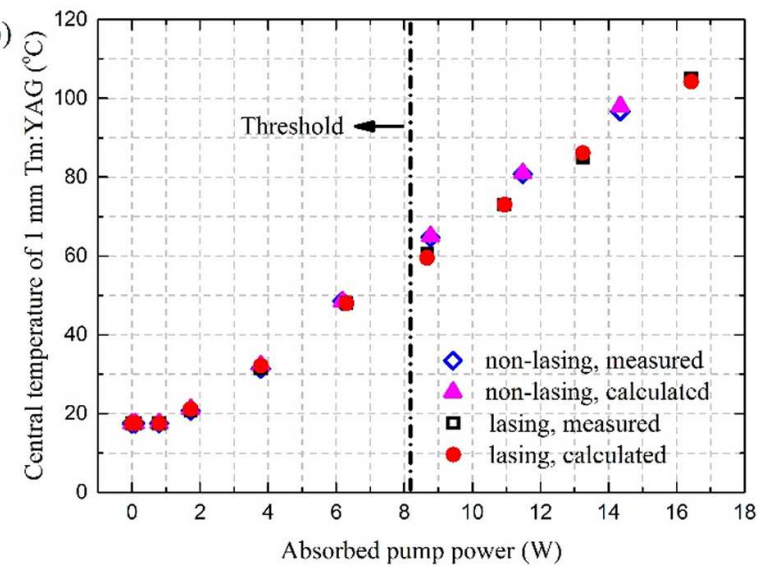

Figure 8. Central temperature of (a) $0.5 \mathrm{~mm}$ Tm:YAG disk and (b) $1 \mathrm{~mm}$ Tm:YAG thick disk versus absorbed pump power under lasing and non-lasing conditions.
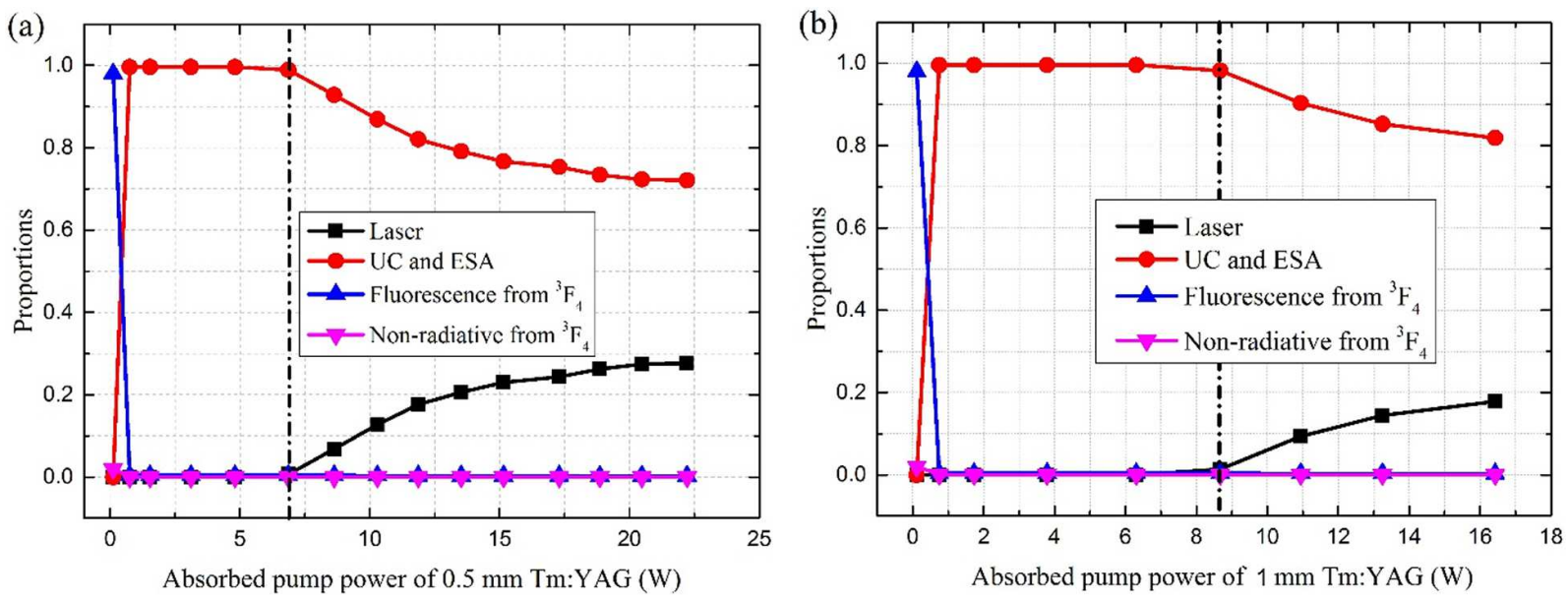

Figure 9. Proportions of all the transitions for (a) $0.5 \mathrm{~mm} \mathrm{Tm}$ :YAG disk laser and (b) $1 \mathrm{~mm}$ Tm:YAG thick disk laser under the lasing condition.

method. The calculated central temperature can be obtained based on the thermal load model. It can be seen that the temperatures of Tm:YAG disk are directly proportional to the absorbed pump power, and are significantly different under lasing and non-lasing conditions. Under lasing condition, the intensities of UC and ESA, denoted by $\left(1-\eta_{L}\right) \eta_{U C \& E S A}$, are decreased due to the increased laser intensity $\eta_{L}$ via SE. As the increased temperature of Tm:YAG is mainly caused by the UC and ESA when pumped by $1 \mu \mathrm{m}$ light ${ }^{[12]}$, the maximum temperature of Tm:YAG is thus decreased from $122.3^{\circ} \mathrm{C}$ to $108.5^{\circ} \mathrm{C}$ at the absorbed pump power density of $2.0 \mathrm{~kW} / \mathrm{cm}^{3}$. Moreover, the central temperature of a $1 \mu \mathrm{m}$ pumped Tm:YAG disk crystal was comparable with the $785 \mathrm{~nm}$ pumped case, where the disk temperature of $150^{\circ} \mathrm{C}$ was reached at an absorbed pump power density of $3.1 \mathrm{~kW} / \mathrm{cm}^{3[10]}$. This is due to the fact that the UC and ESA effects are significant for both $785 \mathrm{~nm}$ and $1 \mu \mathrm{m}$ pumped Tm:YAG disk lasers.

On the other hand, when the absorbed pump power is above the ESA threshold $(0.2 \mathrm{~W}$ of absorbed pump power), the $1 \mathrm{~mm}$ thick disk exhibits approximately
$\eta_{U C \& E S A}=99.7 \%$ and $\eta_{R}^{1 G 4}=0.2 \%$ by using the same method. As the heat dissipation of the $1 \mathrm{~mm}$ thick disk is lower than the $0.5 \mathrm{~mm}$ disk, the temperature of the $1 \mathrm{~mm}$ thick disk is much higher than in the $0.5 \mathrm{~mm}$ case at the same absorbed pump power, as shown in Figure 8(b). At the input pump power of $38.1 \mathrm{~W}$, the central temperature of the gain medium has increased by $50 \%$ from $70^{\circ} \mathrm{C}$ to $105^{\circ} \mathrm{C}$. In addition, the intensity of UC and ESA for the $1 \mathrm{~mm}$ thick disk is higher than in the $0.5 \mathrm{~mm}$ case at the same absorbed pump power, and this is attributed to the weaker intensity of $\mathrm{SE}$ for the $1 \mathrm{~mm}$ thick disk, as shown in Figure 6.

To clarify the dynamic variation of UC and ESA intensities in Tm:YAG disk lasers, the proportions of all heating factors were calculated, as shown in Figure 9. When the absorbed pump power is below the ESA threshold $(0.1 \mathrm{~W}$ for the $0.5 \mathrm{~mm}$ disk case or $0.2 \mathrm{~W}$ for the $1 \mathrm{~mm}$ disk case), the proportions of laser, UC, and ESA are zero. The fluorescence and non-radiative decay from the ${ }^{3} \mathrm{~F}_{4}$ state are dominant with values of $98 \%$ and $2 \%$, respectively. At the same time, the fractional thermal loads of fluorescence and non-radiative decay from the ${ }^{3} \mathrm{~F}_{4}$ state are $5.48 \%$ and $2 \%$, respectively, 

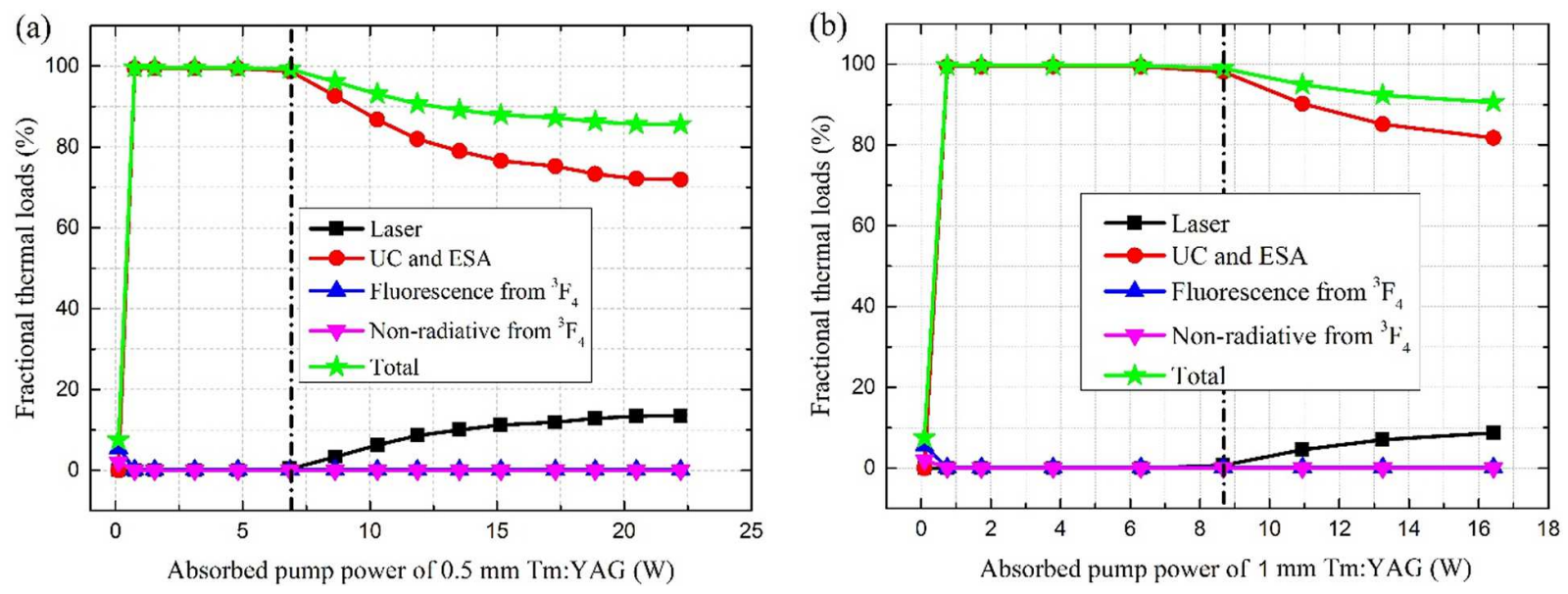

Figure 10. Fractional thermal loads of all transitions for (a) $0.5 \mathrm{~mm}$ Tm:YAG disk laser and (b) $1 \mathrm{~mm}$ Tm:YAG thick disk laser under the lasing condition.

as shown in Figure 10. When the absorbed pump power is above the ESA threshold, the effects of fluorescence and non-radiative decay from the ${ }^{3} \mathrm{~F}_{4}$ state are quite weak. For the $0.5 \mathrm{~mm}$ thickness of gain medium, when the pump power density is lower than the laser threshold, the proportion of UC and ESA effects is $99.6 \%$. This is due to the fact that the absorbed pump power density at this stage is much higher than theoretically required for the onset of significant $\mathrm{UC}$ and $\mathrm{ESA}^{[24]}$. Therefore, the UC and ESA effects are extremely strong. Based on above analysis, the radiative quantum efficiency for the ${ }^{1} \mathrm{G}_{4}$ state $\eta_{R}^{1 G 4}$ is only $0.3 \%$, indicating that the UC- and ESA-induced heating in the Tm:YAG disk laser is mainly through non-radiative decay. As shown in Figure 10(a), the fractional thermal load of the gain medium is over $99 \%$. When the absorbed pump power is higher than the laser threshold, the UC and ESA effects are partly suppressed by the increased laser intensity. The UC- and ESA-induced heating is decreased accordingly. In addition, the total fractional thermal load of the gain medium is also decreased, resulting in the temperature of the Tm:YAG disk dropping after lasing, as shown in Figure $8(\mathrm{a})$. With the further increase of the absorbed pump power, the competition between laser, UC, and ESA will tend to be balanced because of the saturation of optical-to-optical efficiency.

On the other hand, the tendencies of the $1 \mathrm{~mm}$ Tm:YAG thick disk are similar to the $0.5 \mathrm{~mm}$ case over the range of absorbed pump power. At the absorbed pump power of $16.5 \mathrm{~W}$, the laser proportion of the $1 \mathrm{~mm}$ thick disk is $19 \%$, which is lower than that of the $0.5 \mathrm{~mm}$ disk, in which a laser proportion of $24 \%$ is delivered. Conversely, compared with the $0.5 \mathrm{~mm}$ disk, the proportion of UC and ESA for the $1 \mathrm{~mm}$ Tm:YAG disk increases from $75 \%$ to $82 \%$, indicating that the $1 \mathrm{~mm}$ Tm:YAG disk has higher UC- and ESA-induced heating, thereby a higher total fractional thermal load. The higher heat generation, as well as poorer heat dissipation capacity, makes the temperature of the $1 \mathrm{~mm}$ thick gain medium much higher than that of the $0.5 \mathrm{~mm}$ case at the same absorbed pump power, as shown in Figure 8. Therefore, we expect that the Tm:YAG disk with lower thickness and better heat dissipation capacity can achieve higher power and efficiency via this scheme.

\section{Conclusion}

In summary, we have reported a $2 \mu \mathrm{m}$ Tm:YAG disk laser pumped by a $1 \mu \mathrm{m}$ laser via a 20-pass pumping scheme. This scheme enables the evaluation of absorption, output, and temperature properties. The competitive transitions of UC and ESA particularly challenge the scaling of output powers for $2 \mu \mathrm{m}$ disk lasers. To analyze the effects of UC and ESA, a numerical model accounting for SE, fluorescence, non-radiative decay, UC, and ESA has been developed. More broadly, the proportions and fractional thermal loads of all transitions have been derived quantitatively based on the model. Experimental and theoretical results show that the UC and ESA effects are dominant in $1 \mu \mathrm{m}$ pumped Tm:YAG disk lasers, leading the absorbed pump power to decrease abnormally after lasing. The strong UC and ESA are the main sources of total heat generation of the gain medium. In addition, although the Tm:YAG disk with small thickness has a lower absorbed pump power, its higher heat dissipation capacity enables the relatively weak UC and ESA and low total fractional thermal load, which are conducive to the promotion of laser performance. As a result, the $0.5 \mathrm{~mm}$ Tm:YAG disk has a maximum output power of $1.05 \mathrm{~W}$, limited only by the available pump power, and the measurement of it yielded $M_{x}^{2}=2.02, M_{y}^{2}=2.03$. Finally, this model can be used for any $\mathrm{Tm}^{3+}$-doped disk laser, which lays the groundwork for analyzing the operation characteristics and evaluating the dynamic processes of all transitions for diskshaped $\mathrm{Tm}^{3+}$-doped lasers. Further reducing the thickness of the gain medium, together with increasing pump number and spot size, will be propitious to scaling the $\mathrm{Tm}^{3+}$ disk lasers' 
output power without compromising beam quality and mode stability in the near future.

\section{Acknowledgments}

This work was supported by the National Natural Science Foundation of China (NSFC, No. 61975060) and the Fundamental Research Funds for the Central University (HUST, No. 2016YXMS213).

\section{References}

1. I. Mingareev, F. Weirauch, A. Olowinsky, L. Shah, P. Kadwani, and M. Richardson, Opt. Laser Technol. 44, 2095 (2012).

2. B. Wolter, M. G. Pullen, M. Baudisch, M. Sclafani, M. Hemmer, A. Senftleben, C. D. Schröter, J. Ullrich, R. Moshammer, and J. Biegert, Phys. Rev. X 5, 021034 (2018).

3. F. Silva, S. M. Teichmann, S. L. Cousin, M. Hemmer, and J. Biegert, Nat. Commun. 6, 6611 (2015).

4. C. T. Wu, M. X. Yao, T. Y. Dai, and Y. L. Ju, Optik 140, 356 (2017).

5. X. Wang, P. Zhou, X. Wang, H. Xiao, and L. Si, High Power Laser Sci. Eng. 1, 123 (2013).

6. P. Liu, L. Jin, X. Liu, H. Huang, and D. Shen, Appl. Opt. 55, 2498 (2016).

7. A. Diening, B.-M. Dicks, E. Heumann, G. Huber, A. Voss, M. Karszewski, and A. Giesen, in Conference on Lasers and Electro-Optics (1998), paper CWF47.

8. A. Giesen and J. Speiser, IEEE J. Sel. Top. Quantum Electron. 13, 598 (2007).
9. W. L. Gao, J. Ma, G. Q. Xie, J. Zhang, D. W. Luo, H. Yang, D. Y. Tang, J. Ma, P. Yuan, and L. J. Qian, Opt. Lett. 37, 1076 (2012).

10. J. Zhang, F. Schulze, K. F. Mak, V. Pervak, D. Bauer, D. Sutter, and O. Pronin, Laser Photon. Rev. 12, 1700273 (2018).

11. P. B. Phua, K. S. Lai, R. F. Wu, Y. L. Lim, and E. Lau, Opt. Lett. 25, 61 (2000).

12. Y. Chen, H. Wang, J. Dong, M. Wang, H. Chen, Y. Qian, G. Zhu, K. Alekse, and X. Zhu, IEEE Photon. Technol. Lett. 31, 1139 (2019).

13. H. L. Hong, Q. Liu, L. Huang, and M. L. Gong, Laser Phys. Lett. 10, 025002 (2013).

14. C. Vorholt and U. Wittrock, in Conference on Lasers and Electro-Optics, (2015), paper CAP10.

15. Y. Huang, X. Zhu, G. Zhu, J. Shang, H. Wang, L. Qi, C. Zhu, and F. Guo, Opt. Express 23, 4605 (2015).

16. C. Li, Y. Huo, S. He, and Y. Cao, Acta Opt. Sin. 19, 243 (1999).

17. G. Jia, C. Tu, J. Li, Z. Zhu, Z. You, Y. Wang, and B. Wu, High Power Laser Part. Beams 17, 138 (2005)

18. J. A. Caird, L. G. De Shazer, and J. Nella, IEEE J. Quantum Electron. QE-11, 874 (1975).

19. X. Liu, H. Huang, H. Zhu, Y. Wang, L. Wang, D. Shen, J. Zhang, and D. Tang, Opt. Commun. 332, 332 (2014).

20. S. Toroghi, A. K. Jafari, and A. H. Golpayegani, IEEE J. Quantum Electron. 46, 871 (2010).

21. Y. Niu, D. Man, C. Wang, W. Liu, and H. Niu, Proc. SPIE 9266, 92660N (2014).

22. S. Chénais, F. Balembois, F. Druon, G. Lucas-Leclin, and P. Georges, IEEE J. Quantum Electron. 40, 1217 (2004).

23. M. R. Volkov, I. I. Kuznetsov, and I. B. Mukhin, IEEE J. Quantum Electron. 54, 1700106 (2018).

24. S. D. Jackson and T. A. King, IEEE J. Quantum Electron. 5, 779 (1998). 